\title{
Retraction Note to: Letter: On a Semi-Numeric Method to Study the Evolution of a General-Relativistic Radiating Shell and X-Ray Bursters
}

\section{Roberto Aquilano $0^{1,2,3}$}

Published online: 28 May 2021

(C) Springer Science+Business Media, LLC, part of Springer Nature 2021

\section{Correction to: General Relativity and Gravitation, Vol. 35, No. 9, September 2003, https://doi.org/10.1023/A:1025795521614}

The Editors-in-Chief have retracted this article because it significantly overlaps with previous submitted/published articles [1,2].

The author agrees to this retraction.

\section{References}

1. Santoro, F., Aquilano, R., Ibaceta, D.: Contraction of radiating shell in classical and post-Newtonian approximation. Astrophys. Space Sci. 268, 469-474 (1999). https://doi.org/10.1023/A:1002035223 803

2. Monton, C., Scancich, M., Aquilano, R.: Astrophysics of radiating shells. Astron. Astrophys. Trans. 22(3), 357-361 (2003). https://doi.org/10.1080/1055679031000091760

Publisher's Note Springer Nature remains neutral with regard to jurisdictional claims in published maps and institutional affiliations.

The original article can be found online at https://doi.org/10.1023/A:1025795521614.

Roberto Aquilano

aquilano@ifir.iflr.edu.ar

1 Instituto de Fisica Rosario (CONICET - U.N.R.), Bv. 27 de febrero 210 bis, 2000 Rosario, Argentina

2 Facultad de Ciencias Exactas, Ingeniería Y Agrimensura, U.N.R., Av. Pellegrini 250, 2000 Rosario, Argentina

3 Observatorio Astronómico Y Planetario Municipal, Parque Urquiza, Casilla de Correo 606, 2000 Rosario, Argentina 